\title{
Proceso de Bolonia (VI): aprendiendo comunicación para la salud en el Grado de Medicina
}

Francesc Borrell-Carrió, Xavier Clèries, David Paredes-Zapata, Josep Maria Borrás-Andrés, Mireia Sans-Corrales, Joan Josep Mascort-Roca

Resumen. La comunicación forma parte de la actividad clínica y en ocasiones determina el éxito o fracaso del proceso asistencial. Sin embargo, no ha sido reconocida en los planes de estudio del Estado español hasta muy recientemente, sobre todo con el impulso del llamado 'Plan Bolonia'. En el año 2009 se aprobó en la Facultad de Medicina de la Universitat de Barcelona un nuevo plan docente que proponía incorporar la enseñanza de la comunicación en las materias clínicas y preclínicas, contabilizando entre 3 y 6 créditos europeos (ECTS), como competencia transversal. El presente artículo aborda el diseño de esta competencia, los pros y contras, cómo se inserta en diferentes asignaturas y cómo puede evaluarse.

Palabras clave. Comunicación. Enseñanza del Grado de Medicina. Métodos docentes.

\section{Bologna Process (VI): learning health communication in medical undergraduate}

Summary. Communication is part of the clinical activity and sometimes determines the healthcare success or failure. However it has not been recognized in the Spanish curricula until recently, in the context of the so called Plan Bologna. In 2009 a new teaching plan was approved at Medical School of Barcelona University which intended to incorporate communication in clinical and preclinical areas (3-6 ECTS). In this article we explain pros and cons to introduce communication not as a subject, but as a competency, how can be integrated in different subjects and how can be evaluated.

Key words. Communication. Teaching methods. Undergraduate medical education.

\section{Punto de partida}

La comunicación constituye una de las partes nucleares de la competencia de la profesión médica [1-8] y por esta razón se considera susceptible de ser enseñada, aprendida e, incluso, evaluada. En este sentido, el objetivo que nos proponíamos es que los alumnos del Grado de Medicina finalizaran sus estudios con una idea clara de la importancia de la comunicación en todos los ámbitos de su actividad profesional y conociendo los conceptos centrales en esta materia. A tal efecto, el nuevo plan de estudios de la facultad del año 2009 [9] optó por destinar de 3 a 6 créditos europeos (ECTS) a dicha materia. Sin embargo, estos créditos no se reservaban en forma de asignatura, sino en forma de competencia transversal. El matiz es muy importante: si el plan de estudios hubiera constituido una asignatura que se hubiera denominado 'Comunicación en el ámbito de la medicina', con una carga lectiva de 3 a 6 ECTS, hubiera tenido que destinar unos profe- sores y restar estos créditos a otras materias. La opción que se tomó fue, en cambio, nombrar dos coordinadores en esta competencia transversal. Estos coordinadores tenían el encargo de diseñar un módulo de aprendizaje que, a lo largo del Grado de Medicina, diera respuesta a las necesidades formativas que se habían recogido en el plan de estudios.

Esta estrategia es novedosa en nuestra facultad (como lo sería para la mayoría de facultades de medicina) y supone en la práctica lo siguiente:

- No existe una asignatura ni unos exámenes específicos incorporados al curso académico que se denominen 'Comunicación en el ámbito de la medicina'. Los contenidos de esta competencia se incorporan a diferentes asignaturas clínicas a lo largo del currículo y cada asignatura los valora como una de las competencias transversales que enseña. Así, por ejemplo, en semiología se valora no sólo que el alumno conozca las bases conceptuales de una anamnesis clínica, sino los aspectos de comunicación implicados en dicha anamnesis.
Facultad de Medicina; Universitat de Barcelona; Departamento de Ciencias Clínicas (F. Borrell-Carrió, J.M. Borrás-Andrés, M. Sans-Corrales, J.J. Mascort-Roca). Campus Casanovas (D. Paredes-Zapata). Parc Sanitari Pere Virgili (X. Clèries). Barcelona, España.

Correspondencia: Dr. Francesc Borrell Carrió. CAP Cornellà. Bellaterra, 39-41. E-08940 Cornellà de Llobregat (Barcelona).

E-mail:

fborrell.cp.ics@gencat.cat

Agradecimientos:

Editorial Elsevier y Fundación Ciencias de la Salud, que han colaborado generosamente en esta iniciativa docente.

Conflicto de intereses: No declarado.

Conflict of interests: None declared.

(c) 2012 Educación Médica 
- Aunque esta materia no se constituye en asignatura, sí se conforma como módulo de competencia transversal. Como tal módulo, los coordinadores establecen un diseño curricular, unos profesores colaboradores y una estrategia de valoración que es aprobada por la Junta de Facultad.

- El plan de estudios desea mejorar las habilidades comunicativas en diferentes áreas: ámbito de atención médica (comunicación médico-paciente-familia), ámbito del trabajo en equipo (comunicación con otros profesionales y entre profesionales) y, finalmente, la esfera social (comunicar a los medios y a la sociedad).

En este planteamiento se han tenido en cuenta las bases conceptuales y metodológicas más significadas en el ámbito de la educación médica respecto al aprendizaje de comunicación de los futuros profesionales de la salud $[10,11]$. En concreto, cabe señalar el consenso que acordaron las 33 facultades de medicina del Reino Unido sobre las habilidades de comunicación que deben adquirir los estudiantes de medicina en su formación curricular. Destaca que el dominio de comunicación nuclear consiste en el 'respeto a los demás' y que todo el programa formativo se basa en los principios de: profesionalismo, práctica basada en pruebas, aspectos éticos y legales y práctica reflexiva. Cada facultad de medicina adapta los diseños formativos en función de las lagunas apreciadas en su plan de estudios [12].

\section{Estrategia docente}

Las opciones que hemos expuesto de manera sucinta tenían fortalezas y debilidades. La principal fortaleza consistía en legitimar la comunicación ante los alumnos, que podían percibir una asignatura con este nombre como una asignatura 'menor'. En cambio, introduciéndola en medicina interna, oncología, cardiología, etc., trasladábamos el prestigio de estas materias a la competencia de comunicación. La comunicación dejaba de ser vista como una 'isla ideal' donde unos médicos próximos a la 'santidad' se interesaban por los pacientes en tanto que personas.

La principal debilidad era la disimilitud en las actitudes y conocimientos sobre esta materia de los propios profesores encargados de impartirla. Otra debilidad consistía en que el alumno percibiera la unidad conceptual de la competencia, problema que no se suscita cuando tiene que afrontar un examen.

Para paliar en lo posible estas debilidades nos propusimos varias acciones y estrategias:
- Definir un glosario con todos los conceptos y términos que debía aprender el alumno a la finalización del grado. De esta manera lográbamos varios objetivos secundarios del mayor interés: estabilizar un cuerpo teórico de conocimientos, homogeneizar el uso de términos entre los profesores y facilitar que los diferentes profesores que participarían tuviesen una referencia de lo que en otros momentos curriculares se explicaría a los alumnos.

- Ofrecer un workshop anual de formación a los profesores que participan en la competencia. Este workshop tiene por objetivo fundamental explorar el glosario y proporcionar herramientas didácticas.

- De manera personalizada con cada uno de los profesores ofrecerles soluciones 'a medida' para que incorporaran contenidos de comunicación a su asignatura, de acuerdo con el diseño del plan de estudios y el desarrollo posterior aprobado por la Junta de Facultad. En la tabla I recogemos este diseño básico.

Puede resultar interesante para el lector reseñar algunas estrategias didácticas más concretas:

- En ningún caso se ha planteado la colaboración de los diferentes responsables de asignaturas como obligatoria. Siempre, sobre esta base de voluntariedad, nos hemos acomodado a las actitudes y habilidades de cada profesor y responsable de asignatura.

- De manera más concreta para cada asignatura hemos proporcionado los siguientes materiales, siempre en un diálogo fluido con los responsables: diseño y diapositivas (Powerpoint) de las clases o seminarios; clips de vídeo, constituyendo un repositorio común a la competencia (en convenio con Elsevier), y ejercicios para los alumnos.

La comunicación humana sólo se puede aprender si los estudiantes adquieren nuevas formas de integrar y reflejar experiencias personales. La estrategia de enseñanza, por consiguiente, se basa en los problemas clínicos:

- Diferentes maneras de resolver problemas de comunicación y conflictos.

- Decisiones compartidas con el paciente.

- Identificación de errores comunes en el ámbito de la comunicación asistencial.

- Hacer consciente el hábito de comunicación que el alumno tiene de manera habitual, y la gestión emocional que debemos aprender a realizar en la entrevista clínica. Seguimos a tal efecto los modelos que reseñamos en las tablas II y III. 
Tabla I. Módulo interdisciplinario de comunicación y relación asistencial (MICRA) a.

\begin{tabular}{cl} 
Módulo A & $\begin{array}{l}\text { Asociado a psicología y a introducción de la salud. } \\
\text { Contiene conocimientos básicos de comunicación } \\
\text { humana (1 ECTS) }\end{array}$ \\
\hline Módulo B & $\begin{array}{l}\text { Asociado a semiología y otros cursos clínicos } \\
\text { (oncología, enfermedades infecciosas, situaciones } \\
\text { críticas de atención de emergencia). Este módulo } \\
\text { contiene habilidades de comunicación aplicados } \\
\text { al trabajo con pacientes, familia y equipo (1 ECTS) }\end{array}$ \\
\hline Módulo C $\quad \begin{array}{l}\text { Asociado a medicina familiar (6.- curso). Este módulo } \\
\text { explora la resolución de los retos actuales en la } \\
\text { práctica de la medicina, tanto en atención primaria } \\
\text { como en urgencias (1 ECTS) }\end{array}$
\end{tabular}

a Se compone de tres módulos que se desarrollarán durante el Grado de Medicina, con 3 ECTS. Estos módulos se complementan con un curso optativo denominado 'Medicina centrada en el paciente' (2 ECTS) [13].

\section{Proceso didáctico}

Las personas creemos en general que nos comunicamos bien. Algunas personas, pocas, perciben que pueden mejorar aspectos de la comunicación, e incluso algunas se lo toman muy en serio y de manera natural adquieren un alto grado de habilidades sociales. Estas personas aprenden que es muy ventajoso adquirir este tipo de habilidades y se mostrarán receptivas a aprender más. Por consiguiente, cuando un profesor introduce la competencia de comunicación se encuentra con estos perfiles:

- 'Yo ya me comunico bien, no creo que me aporte nada'.

- 'A ver qué me aporta sobre lo que ya he reflexionado'.

- No participar.

Una manera en que el profesor puede afrontar positivamente los dos primeros perfiles es plantear al inicio de la actividad una situación clínica en la que se produce un problema o conflicto relacionado con la comunicación. Estas situaciones ponen de relieve al primer grupo de estudiantes que tal vez sí hay un conjunto de habilidades que aprender, y al segundo grupo invita a pensar cómo aplicarían lo que ya saben. El tercer grupo, formado por aquellos alumnos que no participan de la actividad docente, quedan limitados al aprendizaje vicario en las prácticas clínicas y a la influencia de sus colegas en el pregrado. Este grupo de alumnos debe ser considerado también en el momento de la evaluación de la competencia.
Tabla II. Competencia emocional en la entrevista clínica $[13,14]$.

\begin{tabular}{ll}
\hline $\begin{array}{l}\text { Estilo emocional } \\
\text { 'natural' }\end{array}$ & $\begin{array}{l}\text { El profesional se comporta de manera similar a como actúa en sus relaciones } \\
\text { sociales: conductualmente, con el grado de cordialidad y respeto que } \\
\text { le caracteriza, y emocionalmente, de forma habitual con estilo reactivo } \\
\text { (simpático con el que me cae bien, antipático con el que me cae mal) }\end{array}$ \\
\hline Estilo emocional & $\begin{array}{l}\text { E conocer sus niveles basales, y adopta un estilo emocional proactivo: } \\
\text { profesional básico }\end{array}$ \\
$\begin{array}{l}\text { En el ámbito cognitivo, el profesional sabe establecer unos objetivos básicos } \\
\text { del encuentro, adopta un plan de entrevista y sabe ejecutar unas tareas de } \\
\text { entrevista clínica }\end{array}$ \\
\hline $\begin{array}{l}\text { Estilo emocional } \\
\text { profesional avanzado }\end{array}$ & $\begin{array}{l}\text { o reacciones conductuales y sabe realizar maniobras de rescate emocional } \\
\text { y rescate temático y de atención, entre otras habilidades. En el ámbito } \\
\text { cognitivo, el profesional sabe analizar el encuentro de manera diacrónica } \\
\text { (aplicando un modelo de interacción estratégica), sabe encajar pérdidas de } \\
\text { autoestima y adaptar las conductas lex artis a las peculiaridades concretas } \\
\text { del paciente, con una consideración ética }\end{array}$ \\
\hline
\end{tabular}

Si nos fijamos en cómo trabajar el escenario 'problema, cabe decir que a un escenario inicial pueden seguir otros escenarios representativos de la dificultad que tratamos de superar. Estos escenarios recomendamos trabajarlos siguiendo estos pasos [17]:

- Mostración. Situamos a los alumnos en el contexto en que se produce el reto de comunicación. A continuación les presentamos una escena videograbada (preferible) o un diálogo escenificado o que leemos.

- Reflexión. El profesor tiene preparada una serie de preguntas o comentarios para suscitar la reflexión de los alumnos. Hay que evitar la tendencia a divagar o sugerir actuaciones abstractas, y en cambio orientar a los alumnos a las palabras o acciones concretas que se deberían llevar a cabo.

- Resolución. Se reproduce la escena, esta vez con los propios participantes en el taller o seminario. El profesor lleva a cabo el rol de profesional y pone en práctica las diversas sugerencias de los alumnos. Estas sugerencias a veces no son consistentes unas con otras, por lo que interesará en ocasiones realizar varias escenificaciones para que los alumnos verifiquen cuál es la habilidad de comunicación que logra mejor resultado.

- Práctica personal. Una vez el profesor sintetiza lo que el grupo ha aprendido, se propone a los alumnos que reproduzcan un escenario similar. En grupos de tres, uno asume el rol de paciente, otro, el de profesional, y un tercero, el de observador. Se proporciona una guía de observación -conocida por todos- para este último. Hemos llamado a esta metodología 'ejercicios de $3 \times 3$ '. 
Tabla III. Modelo emotivo-racional de acto clínico [15].

El acto clínico está presidido por la tensión psicológica de dar una respuesta eficaz y eficiente a la demanda del paciente. Una falta de contención de esta tensión psicológica lleva al cierre prematuro de la entrevista, una de las principales causas de errores médicos

Encuadre de la entrevista: comprender con exactitud esta demanda suele ser la primera prioridad del acto clínico. A tal efecto deberemos distinguir entre la expectativa del paciente y lo que el profesional entiende que es su prioridad en aquel momento clínico

En la entrevista semiológica buscamos datos anamnésicos y de exploración física para orientar los problemas del paciente. La irrupción de emociones fuertes altera el curso de la reflexión, facilitando la aparición de las denominadas 'trampas cognitivas' [16]

Una de las principales habilidades 'emocognitivas' del clínico consiste en reencuadrar las prioridades de la entrevista. Ejemplos de reencuadre serían: 'estoy orientando el problema del paciente como dolor de hombro cuando en realidad aqueja una debilidad muscular generalizada', o bien, 'estoy considerando las quejas de este paciente como si fueran de perfil crónico, pero lo cierto es que se trata de unas molestias nuevas'

Los reencuadres suponen gestionar las emociones sobre todo en dos aspectos: evitar la falsa seguridad clínica (overconfidence) y aprender a demorar la resolución de las entrevistas clínicas (contener la prisa)

- Validación y reafirmación grupal. El grupo se reencuentra y se comentan dificultades y situaciones particulares.

- Trabajo personal. Invitamos a los alumnos a trabajar la temática en la parte de trabajo personal que tiene el crédito. A tal efecto disponemos de varios instrumentos: a) Curso virtual a través de la plataforma Moodle, donde los alumnos pueden escoger entre diversas posibilidades de trabajos optativos. La corrección de estas pruebas se realiza también a través del campus virtual; b) Webquest elaboradas especialmente para el módulo; y c) Curso a distancia de 'cómo dar malas noticias' (en convenio con la Fundación Ciencias de la Salud).

En síntesis, se trata de una orientación didáctica que prioriza los retos de comunicación, usa el que hemos denominado 'motor de tres tiempos' [17] (contexto de las escenas, el profesor se pone a riesgo ante los alumnos y en un tercer tiempo el propio alumno es quien afronta el reto), y los ejercicios con un diseño de observador privilegiado (ejercicios de $3 \times 3$ ). Los diferentes recursos online que hemos mencionado se integrarán en un portafolio que el alumno irá siguiendo a lo largo del currículo.

\section{Evaluación}

No existe una 'nota' que sintetice los avances en esta competencia. Como hemos dicho, esta valoración se integra en la propia de cada asignatura. Sin embargo, la comunicación tendrá al menos un $20 \%$ del peso total de la evaluación clínica objetiva estructurada (ECOE) que se celebrará a finales de sexto curso. Una vez instaurado el portafolio, las hojas de reflexión ofrecerán una oportunidad de mejorar la valoración de la competencia.

\section{A manera de conclusión}

Las habilidades de comunicación pueden (y deben) aprenderse, pero para lograr este objetivo es necesario un primer paso: la comunicación debe ser reconocida por los profesores y estudiantes como parte fundamental del plan de estudios clínicos. En este reconocimiento tiene mucho que ver la actitud que se adopta hacia la comunicación y cómo se entiende ésta. Si la comunicación se contempla sólo como una mera transmisión de información, se pierde la visión global, compleja y contextual del ser humano ante los procesos de salud y enfermedad.

Los coordinadores de esta competencia deben ser legitimados por la Junta de Facultad y gozar del apoyo activo de los responsables académicos, en especial del jefe de estudios. La estrategia docente debe ser participativa, basada en seminarios o talleres, y en problemas o retos que susciten el interés de los alumnos desde el primer minuto. Existe el peligro de que una parte de los alumnos entienda el aprendizaje de la comunicación como unas recetas, eso es, unas habilidades y técnicas que aplicarán a cada situación reto, sin más aprendizaje. Este perfil de alumno debe comprender la parte de gestión emocional que conlleva este aprendizaje. De nada sirve aprender unas habilidades que no se saben enmarcar en una buena gestión de las emociones porque, a largo plazo, será la forma de vivir el acto clínico la que mandará sobre las habilidades adquiridas de manera mecánica. Por si fuera poco, una fuerte emoción de oposición, irritación o antagonismo anula toda posibilidad de empatía [18] y aleja de un abordaje centrado en el paciente.

Bibliografía

1. SEDEM (Sociedad Española de Educación Médica), ACEM (Associació Catalana d'Educació Mèdica), SEMDE (Sociedad de Educación Médica de Euskadi), SADEM (Sociedad Aragonesa de Educación Médica). Recomendaciones para 
un nuevo proceso de reforma curricular en las facultades de medicina españolas. Educ Med 2005; 8: 3-7.

2. Agencia Nacional de Evaluación de la Calidad y Acreditación (ANECA). Libro blanco. Título de Grado en Medicina, Odontología, Enfermería, Psicología y Farmacia. URL: http://www.aneca.es/media/150312/libroblanco_medicina_ def.pdf, http://www.aneca.es/media/150344/libroblanco_ odontologia_def.pdf, http://www.aneca.es/media/150360/ libroblanco_jun05_enfermeria.pdf, http://www.aneca.es/ media/150356/libroblanco_psicologia_def.pdf, http://www. aneca.es/media/150368/libroblanco_farmacia_def.pdf.

3. AQU Catalunya. Competències professionals bàsiques comunes dels llicenciats en Medicina formats a les universitats de Catalunya. Barcelona: Agència per a la Qualitat del Sistema Universitari de Catalunya; 2004. URL: http://www.medab.uclm.es/publico/master/m1/Requeriments \%20globals\%20minims\% 20educacio\% 20medicina-.pdf.

4. General Medical Council. Review of 'Tomorrow's doctors' (2008). URL: http://www.gmc- uk.org/news/articles/ Review\%20of\%20Tomorrow.pdf.

5. The Scottish Dean's Medical Education Group. The Scottish doctor. Learning outcomes for the medical undergraduate in Scotland: a foundation for competent and reflective practitioners. 3 ed. Dundee: Association for Medical Education in Europe (AMEE); 2008.

6. Swing SR. The ACGME outcome project: retrospective and prospective. Med Teach 2007; 29: 648-54.

7. Hamilton JD. International standards of medical education: a global responsibility. Med Teach 2008; 22: 547-8.

8. Clèries X. La esencia de la comunicación en educación médica. Educ Med 2010; 13: 25-31.
9. Facultat de Medicina. Pla d'Estudis del Grau de Medicina. Universitat de Barcelona (2009). URL: http://www.ub.edu/ web/ub/ca/estudis/oferta_formativa/graus/fitxa/M/G1046/ pladestudis/index.html.

10. Makoul G. Essential elements of communication in medical encounters: the Kalamazoo consensus statement. Acad Med 2001; 76: 390-3.

11. Brown J. Perspective: clinical communication education in the United Kingdom: some fresh insights. Acad Med 2012; 87: 1101-4.

12. Von Fragstein M, Silverman J, Cushing A, Quilligan S, Salisbury H, Wiskin C; UK Council for Clinical Communication Skills Teaching in Undergraduate Medical Education. UK consensus statement on the content of communication curricula in undergraduate medical education. Med Educ 2008; 42: 1100-7.

13. Borrell F. Práctica clínica centrada en el paciente. Madrid: Triacastela; 2012.

14. Borrell F. Competencia emocional del médico. FMC 2007; 14: 133-41. URL: http://issuu.com/javipache/docs/ competencia-emocional.

15. Borrell-Carrió F, Epstein RM. Preventing errors in clinical practice. A call for self-awareness. Ann Am Med 2004; 2: 310-6.

16. Croskerry P. Clinical cognition and diagnostic error: applications of a dual process model of reasoning. Adv Health Sci Educ Theory Pract 2009; 14 (Suppl 1): 27-35.

17. Borrell F. Entrevista clínica. Manual de estrategias prácticas. Barcelona: SEMFYC; 2004

18. Borrell F. Empatia, valor troncal en la pràctica clínica. Med Clin (Barc) 2011; 136: 390-7. 